\title{
A Validated Computer Terminology Test for Predicting Non-native English-speaking CS1 Students' Academic Performance
}

\author{
Suad Alaof* \\ salaofi@su.edu.sa \\ University College Dublin \\ Dublin, Ireland
}

\author{
Seán Russell \\ Sean.Russell@ucd.ie \\ University College Dublin \\ Dublin, Ireland
}

\begin{abstract}
This research examines the validity and efficacy of an English computer terminology multiple-choice test. The test was developed to investigate the relationship between non-native English-speaking students' knowledge of computer terminology and their academic performance in CS1 courses. In this research, the Rasch model was used to find empirical evidence of test validity. Six aspects of construct validity were evaluated: content, substantive, structural, generalisability, external and consequential aspects. Based on analyses of 150 students' responses, the test shows an acceptable level of validity in all six aspects of construct validity. Moreover, the efficacy of using this test to predict the students' academic performance in CS1 courses was investigated. Statistical analysis of the data shows that the computer terminology test can predict approximately $50 \%$ of final exam performance in CS1 courses after controlling the impact of English proficiency. The aim of this research (along with other ongoing research) is to aid in the identification of non-native English-speaking students who require more specific language support. Additionally, the research will guide the development of resources and tools that can help these students.
\end{abstract}

\section{CCS CONCEPTS}

- Social and professional topics $\rightarrow$ CS1; CS1; Computer science education.

\section{KEYWORDS}

CS1, programming, non-native English-speaking students, predicting

ACM Reference Format:

Suad Alaofi and Seán Russell. 2022. A Validated Computer Terminology Test for Predicting Non-native English-speaking CS1 Students' Academic Performance. In Australasian Computing Education Conference (ACE '22), February 14-18, 2022, Virtual Event, Australia. ACM, New York, NY, USA, 10 pages. https://doi.org/10.1145/3511861.3511876

*Also with Shaqra University.

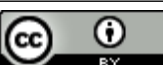

This work is licensed under a Creative Commons Attribution International 4.0 License.

ACE '22, February 14-18, 2022, Virtual Event, Australia

(c) 2022 Copyright held by the owner/author(s).

ACM ISBN 978-1-4503-9643-1/22/02.

https://doi.org/10.1145/3511861.3511876

\section{INTRODUCTION}

The number of international students in Western universities is growing significantly and becoming an important source of funding for these institutions. This results in a larger number of non-native English-speaking (NNES) students in classrooms of Western universities. Additionally, there has been a noticeable increase in the adoption of English medium instruction (EMI) in higher education around the world [30], especially for science disciplines such as computer science (CS). This increasing adoption of EMI internationally is often motivated by the desire of many universities to be globally recognized institutions $[15,26]$.

Both of the above trends result in an increasing number of students globally studying through the medium of English when it is not their native language. The increased cognitive load experienced by these students in EMI classes is linked to both increased apprehension and reduced listening fidelity [12, 45]. Specifically for CS1 students, it has been shown that the use of EMI increases the difficulty of the subject, as students need to understand new programming concepts through a foreign language [33, 42]. Furthermore, this increase in cognitive load could result in low performance in programming courses or exams [18, 20,32]. After comparing the academic performance in programming courses of native and non-native English speaking students, a significant difference was found in favour of those whose native language is English [33, 46].

Keen and Etzkorn [23] conducted a study to investigate the impact of using buzzwords in lecture notes on students' grades. They defined buzzwords as words with CS meaning; however, it is not clear how they selected those words or what those words are. In addition, the study made no distinction between native and non-native English-speaking students, and yet it concluded that the higher the number of difficult terms used in lecture notes, the lower the scores students get in the programming class.

Several studies have compiled lists of the most frequently occurring words in the CS discipline [2, 7, 37]. These lists are aimed at identifying the important words to aid the study of NNES students at different levels. Minshall [37] generated a word list based on the contents of journal articles and conference proceedings in a number of CS disciplines, identifying important words for postgraduate students. Bi [7] compiled a word list based on CS undergraduate textbooks, identifying important words for undergraduates of all levels.

In our previous work, we generated a word list based specifically on textbooks and lecture notes for introductory programming classes, identifying the important words for CS1 students [2]. Based on the CS1 word list, we developed an English Computer Terminology Test (CTT). The CTT is a multiple-choice test designed to 
evaluate CS1 students' level of knowledge in computer terminology [2]. The purpose of this test is to aid in the identification of students who need more support in English computer terminology and consequently improve their academic performance [1].

However, before the CTT can be recommended for wider use, it requires evaluation in terms of its validity and efficacy. Therefore, the first aim of this study is to examine the validity of the test. This research also aims to investigate the efficacy of using this test to predict students' academic performance in CS1 courses.

This paper addresses the following research questions: (1) Is the Computer Terminology Test a valid test of students' level of knowledge of English computer terminology? (2) Can the results of the Computer Terminology Test be used to identify students whose academic performance may suffer as a result of poor knowledge of computer terminology?

Section 2 will discuss the related work with more focus on the use of the Rasch model in test validation. Section 3 describes the methods and instruments used in this research, as well as the participants and sample size. The test validity analysis results are presented and discussed in section 4 . The results of regression analysis performed to investigate the possibility of using the CTT to predict students' academic performance are presented and explained in section 5 . Finally, the conclusion, and limitations and future work are presented in section 6 and section 7, respectively.

\section{BACKGROUND}

In CS1 courses provided through EMI, all learning materials are provided in English regardless of the students' native language [15]. Furthermore, the higher-quality and most up-to-date online resources, such as textbooks, online courses, technical forums, and coding documentation, are usually available in English [20]. Such materials are heavily loaded with computer terminology and concepts that NNES students often find ambiguous and difficult to comprehend [18, 25]. Programming courses, particularly CS1, generally use one of the more mainstream or popular programming languages. The keywords of the majority of these languages have been designed to help learners understand their uses based on their English meaning, which puts NNES students at a disadvantage [33]

Generally, English terminology connotations can differ based on the field in which they are used. This can be a particularly acute problem in CS, as new terms are developed very rapidly [8] Therefore, poor and inaccurate translation often causes more confusion for NNES students, which has a negative impact on their learning [25]. The issue of difficult terminology is not limited to $\mathrm{CS}$, as it has also been raised in other science subjects such as maths, in which NNES students are more likely to struggle because of English mathematical terminology, which often impacts their performance [13, 27].

Moreover, it appears that computer terminology is a factor that has an impact on CS students' performance in any course that uses a language other than the students' native language. A recent study [38], found that non-native Chinese students' performance in the Basics of Computer Application course can be predicted more accurately by their level of knowledge of Chinese computer terminology than their general Chinese language level. The study emphasises the influence of computer terminology on non-native students. Accordingly, the availability of a valid and reliable instrument to measure CS1 students' knowledge of English computer terminology could have a positive impact on CS1 course delivery, especially for NNES students.

The concept of a valid test was first defined by Kelley [24] as a test that measures what it is intended to measure. Messick $[35,36]$ provided a clearer definition of validity, establishing six aspects of validity: content, substantive, structural, generalisability, external and consequential aspects. Smith Jr [47] first described the use of the Rasch model [40] to examine those six aspects. The use of the Rasch model for this purpose over time led to the development of criteria to determine each aspect of validity based on selected mathematical statistics [48]. The application of the Rasch model in this way is supported by the development of software such as Winsteps and Facets that simplify the calculation of these statistics [22].

The content aspect is concerned with the test content's representativeness and its relevance to the construct domain and its technical quality. The substantive aspect relates to finding evidence to prove that test takers are genuinely engaged with the tasks included in the test. The structural aspect of construct validity usually relates to the scoring scheme. The generalisability aspect is concerned with the extent to which the scores' meanings and interpretations can be generalisable to other tasks that are not included in the test but are part of the domain under examination. The external aspect is concerned with the relationships between the test scores and other test and non-test behaviours. Finally, the consequential aspect of construct validity is concerned with the actual and potential consequences of score interpretation on future actions.

The Rasch model was first introduced by mathematician Georg Rasch [40] and is usually described as a prescriptive model since it determines specific conditions that must apply to a test for it to be considered valid. It has proven to be reliable and effective as a framework for verifying the construct validity of multiplechoice tests $[5,44,48]$. The Rasch model has been used in various research fields, including education, medicine, and many others [3]. In education, it is widely used for assessing the validity of measurement and testing instruments in language teaching as well as other subjects [34]. In particular, the Rasch model has been used as a method to evaluate the construct validity of vocabulary tests by many researchers, for example, $[5,6,31,43]$ and many others.

The Rasch model provides several analyses that can be used to find empirical evidence for each aspect of construct validity [9, $47,49]$. Even though the content aspect of construct validity can be determined by the professional judgment of experts [5], it is still possible to evaluate it using the Rasch model. Fit indices can measure the relevance of the test content to the domain under investigation; items that do not fit can be irrelevant to that domain. Furthermore, a person-item map and item strata are very useful in ascertaining the representativeness of the items [5]. The test must include two levels of difficulty to be considered an appropriate representative of the intended domain [47]. However, large gaps in the item difficulty hierarchy reflect that an area of the domain has not been covered [4]. Moreover, the technical quality of the test can be assessed using item-measure correlations since these can highlight poor-quality or mistyped items [49].

Person fit statistics and multiple-choice distractor analysis are very important measures for the substantive aspect of construct 
validity. Person fit statistics measure the extent to which a person's response pattern matches the pattern predicted by the model [47]. Guessing or carelessness in responses shows a misfit in the analysis. Multiple-choice distractor analysis in the Rasch model includes $\mathrm{p}$-values, choice means and distractor-measure correlations. The p-value measures how many times each distractor has been chosen. Since the ideal situation where each distractor is expected to be equally attractive is impossible, $\mathrm{p}$-values are used to detect faulty distractors [49]. Choice means refers to the average of respondents who choose each distractor; it shows its discrimination power. Ideally, distractors should differentiate between high- and low-ability respondents.

Item-measure correlations can also be employed to ensure the unidimensionality of the test [49], which represents the structural aspect of construct validity. In other words, fit statistics can indicate that single scoring is not appropriate, and separate scores should be defined for each dimension. The generalisability aspect of construct validity can be examined by the invariance of item measures across different populations or over time [5]. It is also possible to use the invariance of person measures across different sets of test items. The ability of the test to detect within-individual or between-group changes can determine the external aspect of construct validity [35] Thus, the person-item map and person-strata index are very useful for determining this aspect [50]. The only aspect of construct validity that the Rasch model does not specify a straightforward calculation for is the consequential aspect. However, checking item bias and investigating differential item functionality - or even examining the person-item map more closely - can help to ensure the consequential aspect of construct validity [5].

\section{RESEARCH METHODS}

The main instrument is a multiple-choice test that contains 30 questions about CS1 computer terminology. In each question, a short and simplified definition of a CS1 computer term is given. The students are asked to choose the correct term that matches the definition from three options. No negative marking is applied. The terminology on the test, by question order, includes binary, execution, loop, syntax, data type, iteration, debugging, initialization, variables, Boolean, keywords, identifier, control structure, array/list, integer, exit, algorithm, comma, counter, increment, numeric, compiler, interpreter, parentheses, processor, data structure, module, indentation, function and import/include (for the full test, see appendix A). The process of developing and building this test can be found in our previous work [2].

The test was conducted online and made available to students in the middle of their course. The students were allowed 30 minutes to answer all the test questions; however, most students completed the test in 15 minutes or less. The participants were first-year students studying CS or a closely related discipline, attending CS1 courses conducted mainly in the English language at two universities in different countries. The two institutions are Beijing-Dublin International College (BDIC) in China and Shaqra University in Saudi Arabia. In both institutions, the CS1 course is the first experience of using computer programming to solve computational problems for the students in their undergraduate programmes.
CS1 is generally used as an acronym for the "Introduction to Programming" course; however, there is no worldwide agreement on the exact content of this course [21]. This explains the differences in the structure and topics that these courses cover in this study. In general, most CS1 courses aim to provide an overview of algorithms, algorithm design, and the basics of problem-solving techniques [14]. They also include practical exercises on one of the most popular programming languages.

In this study, both CS1 courses are designed to provide an overview of algorithms and programming languages, understanding and using flowcharts and pseudocode, data types and variables, decisions, repetition, arrays or lists. The CS1 course at BDIC also covers the basics in functions, modular design and files. The CS1 course at Shaqra University uses Java as the main programming language, whereas at BDIC, students learn either $\mathrm{C}$ or Python based on their specific discipline.

The CS1 courses in both institutions are provided mainly in English, which means that all lectures, learning materials, assessments and exams use only the English language. The main difference is that the instructors in Shaqra University are native Arabic speakers (the same language as the students) and they are willing to communicate with the students in their native language if necessary. However, instructors of the CS1 courses in BDIC are native English speakers who do not speak the students' native language and consequently only use the English language to communicate with the students.

In total, 150 NNES students participated voluntarily ${ }^{1}$ in the research study which included taking the CTT test and English proficiency test. Approximately $60 \%$ of the participants were native Chinese speakers and the remaining $40 \%$ were native Arabic speakers. All the participants were aged 18-23 years, 108 were male and 36 were female, while the remaining 7 preferred not to say their gender. Furthermore, about $90 \%$ of the participants stated that they had no previous programming experience.

To investigate test validity, the students' responses were analysed using Winsteps software, version 4.8.2 [29]. Various statistical methods were applied to the data, including item and person fit measures, item and person strata, and others. This process and the results are described in section 4 .

In addition, regression analysis was conducted using SPSS statistics software to explore the efficacy of using the CTT to predict students' academic performance in CS1 courses. The academic performance included three measures: a practical assessment score, a final exam score, and an overall score. In this process, the impact of the students' proficiency in the English language was controlled using their score in a standard English proficiency test [17]. This process and the results are discussed in section 5 .

\section{THE VALIDITY OF THE TEST}

\subsection{Validation Results}

In Rasch model analysis using Winsteps, unexpected response patterns to the test can be detected by INFIT statistics, while OUTFIT

\footnotetext{
${ }^{1}$ We received ethical exemptions for the study conducted in this paper with reference:LS-E-20-129-Alaofi-Russell from University College Dublin and Ethics Appl.202001 from Shaqra University - College of Computing and Information Technology
} 
can detect guessing or mistakes [28]. Fit statistics consist of meansquare values (MNSQ) and z-standardized scores (ZSTD). An MNSQ greater than 1.0 indicates underfit to the model, which means that the data are less predictable than expected. On the other hand, an MNSQ value less than 1.0 indicate overfit to the Rasch model, which means that the data are too predictable. In other words, items with MNSQ values less than 1.0 do not contradict what is already known but do not add anything about what needs to be known, which makes them inefficient. This is why overfit is less important than underfit in test validation measures [28].

Moreover, Winsteps software tests the hypothesis that MNSQ= 1.0, which means that the randomness in the data is equal to the randomness predicted by the Rasch model. The result of this hypothesis testing is presented as ZSTD values. In general, a ZSTD value greater than \pm 1.96 indicates that the null hypothesis of 'model fit' is rejected. For short multiple-choice tests, acceptable values are between 0.7 and 1.3 for MNSQ and between -2.0 and 2.0 for ZSTD for both INFIT and OUTFIT statistics [10].

The validation process for CTT started with item fit statistics; these were analysed for assessing the content and structural aspects of validity. Table 1 provides a summary of item fit statistics for all test measures. The item strata separation shown in the table can be used to assess the content aspect of validity, particularly representativeness. A test should have at least two levels of item difficulty to be considered representative [47]. Clearly, this test has a good level of representativeness, with a separation value of 4.03 . Item reliability stands at .94 , indicating that the sample size was large enough to ensure the item difficulty hierarchy [28]. It is also noticeable that the test has acceptable mean values of INFIT and OUTFIT MNSQ and ZSTD measures.

Table 1: Summary of Item Statistics

\begin{tabular}{llrrrrr}
\hline & & & \multicolumn{2}{c}{ INFIT } & \multicolumn{2}{c}{ OUTFIT } \\
Item & Total & Count & IMNSQ & ZSTD & OMNSQ & ZSTD \\
\hline Mean & 94.5 & 149.9 & 1.00 & .1 & .98 & .0 \\
P.SD & 24.0 & .2 & .11 & 1.5 & .20 & 1.6 \\
\hline \# Input & \multirow{2}{*}{30} & Separation & 4.08 & & Reliability & .94 \\
\hline
\end{tabular}

Figure 1 represents the person-item map of 150 responses to the CTT using Winsteps software [29]. The most unique feature of this map is the placing of persons and items on the same scale which is very useful in comparisons [41]. In other words, the persons' ability and items' difficulty is mapped using unified logit measures derived from the raw score in the analysed data. The measures column on the far left shows the logits units that represent the levels of the students' ability and items' difficulty [28]. The right side on the map shows the test items (Q) arranged by difficulty level from hardest at the top and easiest at the bottom. Similarly, the left side of the map shows the position of the test takers indicated by (X) based on their ability level. Students who demonstrate a higher ability level appear at the top of the figure, and those that demonstrate a lower ability level appear at the bottom. Similarly, the items with high difficulty levels appear at the top of the map and the easy items appear at the bottom.
In Figure 1 the test items show an acceptable distribution against each level of students' abilities. However, Q13 and Q8 are above most students' abilities, whereas Q1 and Q3 are too easy for the tested sample. It is also shown on the left of the map that some students are above the test level; those students obtained results of $90 \%$ or above. The only noticeable gap, although not huge, in the map is between Q26 and Q8, which means that there are no corresponding questions for students with this level of ability. To cover this gap in the test, two questions with medium-to-high level difficulty can be added. In general, it can be said that the test has an acceptable level of representativeness.

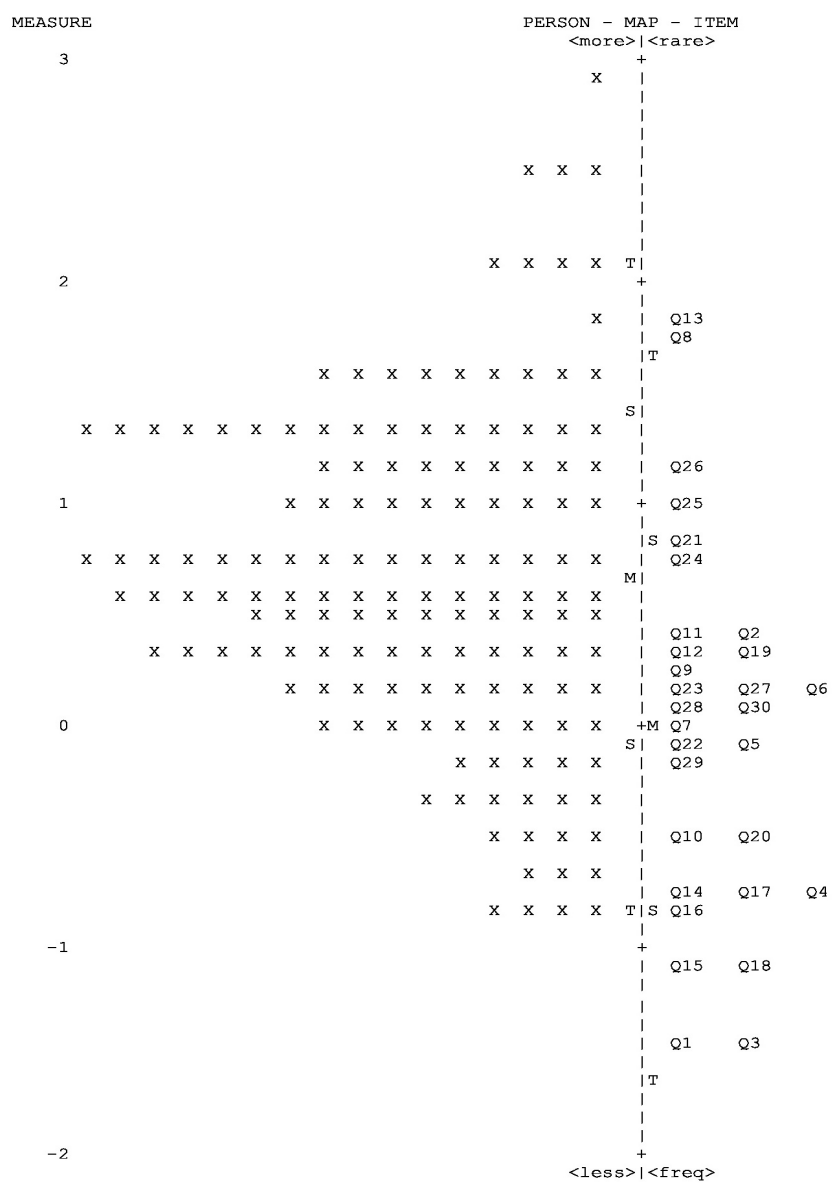

Figure 1: Person-Item Map

The detailed output of item fit statistics has been checked to confirm items' relevance to the intended construct. It confirms that most of the test items are within the acceptable range of MNSQ and ZSTD values. The item fit statistics output for the 30 items of the CTT is too long to be included in this paper. Instead, the items that show values outside the acceptable range of MNSQ and ZSTD have been included in Table 2. Items Q8, Q26, Q21 and Q29 (Table 2) show OUTFIT ZSTD scores of 2.41, 2.17, 2.13 and -2.14, respectively, which are slightly over the acceptable value.

According to Linacre [28], more attention should be given to INFIT indices rather than OUTFIT since they flag misfit items in 
the regions that should be properly covered by the test. Therefore, items Q8, Q26, Q21 and Q29 can be considered a good fit for the test since they show acceptable values in all other statistics. The exceptions here are questions Q13, Q11 and Q6, which represent irrelevant or misfitting items in the test. In general, 27 of the 30 items have fair fit statistic values, indicating an acceptable level of relevance.

Item-measure correlations provide evidence of technical quality (part of the content aspect of validity) and unidimensionality (which represents the structural aspect [11]). It represents the direction of what the item is measuring. Item correlation close to zero means that the item is either very easy, very difficult, or it could be measuring the construct in a different way to other items [49]. On the other hand, a negative correlation indicates that an item is opposing the direction of construct measurements. As mentioned previously, the output of the item measures statistics is too long to be included in this paper. For this reason, only items that show unacceptable values are presented in Table 2. All the other items have been carefully checked, and it has been confirmed that all their measures fell within the acceptable range. Again, Q13 shows a negative correlation (CORR.) in Table 2, indicating a possible technical issue (e.g. ambiguity). Additionally, Q8 and Q11 have correlation values very close to zero. All other items show an acceptable level of correlation, which supports the technical and structural aspects of validity.

To sum up, the content aspect of validity, including relevance, representativeness and technical quality, has been assessed using different types of statistics. Item strata separation and the person item map provided evidence that the test has a good level for representativeness. Moreover, the detailed output of item fit statistics, particularly, MNSQ and ZSTD values of INFIT and OUTFIT, have been investigated to ensure items' relevance. Based on the results, 27 of the 30 questions show an acceptable level of relevance to the construct domain. Finally, Item-measure correlations values for all items have been checked to evaluate the technical quality. The results show that three questions might have some technical issues, whereas the remaining 27 questions are good. Therefore, it can be said that the test has an acceptable degree of relevance, representativeness and technical quality, which supports the content aspect of validity.

Distractor analysis was performed to examine the substantive aspect of validity. This analysis shows the mean of the ability estimates of all test takers who have chosen the correct and wrong options. It is expected that the ability mean of the correct answer will be greater than the wrong answers, otherwise, the distractor is not functioning in the intended way. The output of this analysis is too long to be included; however, the result has been carefully examined. In this analysis, Q13's ability mean values are .48 and .73 for the right and wrong answers, respectively. This indicates that the wrong answer options provided for Q13 are not working correctly. Therefore, this item should be revised or removed from the test. All other items show acceptable values. Thus, the substantive aspect of validity for this test has been confirmed.

\begin{tabular}{llrrrrr}
\hline \multirow{2}{*}{ ITEM } & \multicolumn{2}{c}{ INFIT } & \multicolumn{2}{c}{ OUTFIT } & \multicolumn{2}{c}{ PTMEASUR-AL } \\
& IMNSQ & ZSTD & OMNSQ & ZSTD & CORR. & EXP. \\
\hline Q6 & .85 & -2.63 & .83 & -2.28 & .51 & .31 \\
Q8 & 1.19 & 2.08 & 1.31 & 2.41 & .03 & .31 \\
Q11 & 1.20 & 3.55 & 1.23 & 3.22 & .06 & .32 \\
Q13 & 1.29 & 2.81 & 1.64 & 4.21 & -.15 & .30 \\
Q21 & 1.11 & 1.96 & 1.14 & 2.13 & .18 & .33 \\
Q26 & 1.10 & 1.53 & 1.18 & 2.17 & .17 & .32 \\
Q29 & .86 & -1.96 & .79 & -2.14 & .49 & .30 \\
\hline
\end{tabular}

Table 2: Selected Item Statistics

This test was completed by two groups of students considered to be from different populations due to their native languages. The invariance of item measures across groups has been used to examine the generalisability aspect of validity. The differential item functioning plot in Figure 2 shows that all the item measures are between the control lines. Clearly, the test is valid regarding the generalisability aspect. Moreover, no significant differential test functioning (DTF) was found between groups (Figure 2), meaning that there is no evidence for biased items. Thus, the consequential aspect of validity is ensured, and any decision made based on this test result is expected to be fair.

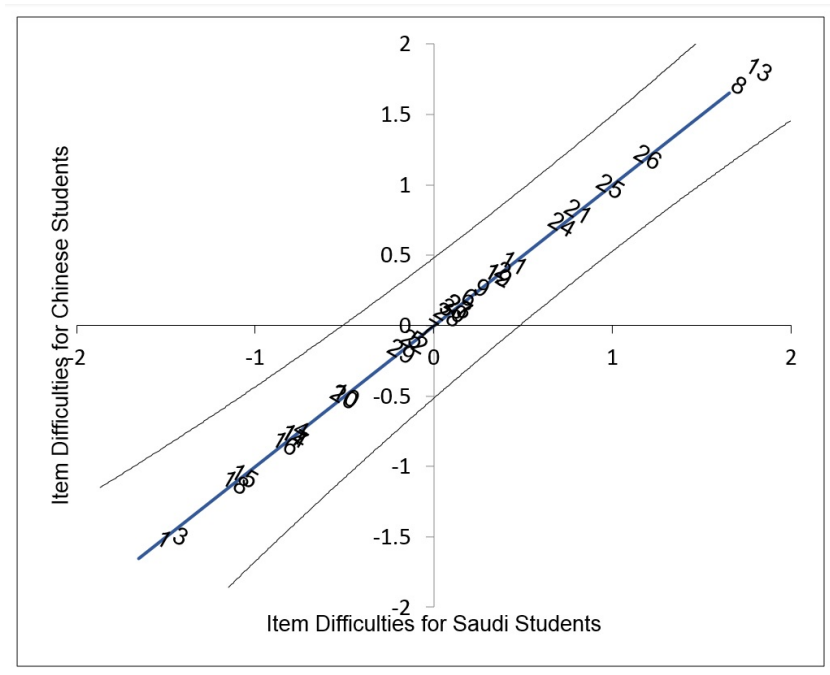

Figure 2: Between-groups differential test functioning

To ensure the external aspect of validity, the person separation strata should be close to 1 [48]. For this test, the person separation stands at 1.30 (Table 3), which is considered an acceptable value. Furthermore, a test that can differentiate between students with low and high levels of abilities can be considered as having external validity [5]. On the person-item map (Figure 1), students with a very high level of ability appear at the top, and students with a low level of ability appear at the bottom, which represents a type of hierarchy in the ability levels. Therefore, it can be said that this test has an acceptable level of differentiation between various levels of students' abilities. 
Table 3: Summary of Person Statistics

\begin{tabular}{llrrrrr}
\hline & & \multicolumn{3}{c}{ INFIT } & \multicolumn{2}{c}{ OUTFIT } \\
Item & Total & Count & IMNSQ & ZSTD & OMNSQ & ZSTD \\
\hline Mean & 18.9 & 30.0 & .99 & .1 & .98 & .0 \\
P.SD & 4.1 & .2 & .19 & 1.2 & .29 & 1.2 \\
\hline \# Input & 150 & Separation & 1.30 & Reliability & .63 \\
\hline
\end{tabular}

\subsection{Validation Summary and Discussion}

In the previous section, the results of the validation analysis of the CTT were presented. This analysis was performed using the Winsteps software [29]. The data used to investigate the test validity were collected through an online test platform. A total of 150 NNES students enrolled in two CS1 courses at different institutions participated in the test. Even though a large sample of participants from different backgrounds was used in this study, the results are still limited to NNES students and the online testing method. Therefore, the validity of this test to determine the computer terminology level of native English speaking students in CS1 has not been explored. Additionally, the impact of providing the test as a paper-based test on the validity results requires further investigation.

Overall, the CTT shows an acceptable level of validity in this study. Regarding the content aspect, $90 \%$ of the test items are relevant to the intended construct based on fit statistics (the exceptions are Q13, Q11 and Q6). It also can be said that the test has a fair level of representativeness based on item separation statistics and the person-item map.

It has been confirmed that the technical quality and structural aspects are within acceptable levels based on an examination of the item-measures correlation except for Q13. All test items show an acceptable substantiveness level based on multiple-choice distractor analysis (again, except for Q13). Furthermore, the invariance of item measures across two different populations confirms the generalisability aspect. Similarly, the differential item functioning shows no evidence of bias, which confirms the consequential aspect.

However, a closer examination of some of the test items, namely, Q13, Q11 and Q6, is needed. The worst case is Q13; its INFIT and OUTFIT measures are high, its item-measures correlation is negative, and its ability mean has an unacceptable value. It also appears at the very top of the person-item map, which means it has a high level of difficulty. Clearly, this question is problematic and should be reviewed or removed from the test.

On the other hand, Q11 and Q6 have only one issue: high ZSTD values in the INFIT and OUTFIT measures. This indicates that those questions measure something other than the intended content, and therefore they should be revised [5]. In addition, Q8 has a high ZSTD OUTFIT value, a near-zero item-measure correlation, and a high level of difficulty based on the person-item map. However, as stated by Lincare [28], a misfit OUTFIT value is not a large problem because of the test's sensitivity to guessing and carelessness. The low correlation measure could be a result of the high level of difficulty. Based on this fact, Q8 can be considered the most difficult question on the test. However, this question can still be a good fit if the test sample represents a higher level of ability; therefore, there is no need to remove this item from the test.
Recommendations to enhance the test can be made based on the person-item map. As Q1 and Q3 tend to be very easy, they could be revised or replaced to give the test a higher level of difficulty. However, this is not recommended if the test will be provided to students who have no basic knowledge of computer science. Additionally, more items with a medium-to-high level of difficulty can be added to expand the ability levels that the test covers. However, the test's new duration and length should be considered if more questions are added. Furthermore, it is very important to consider the scheduling of the test, as giving this test in the first few weeks of a CS1 course, for example, will make it more difficult compared to giving it in the middle or at the end of the course.

These results address the first research question in that the CTT can be considered a valid test of NNES students' level of knowledge of English computer terminology. This is based on the assumption that a modified version is used where question 13 is removed or altered to address the issues identified in the analysis. Moreover, it is important to note that the use of a paper-based testing method may yield different results.

\section{PREDICTION OF ACADEMIC PERFORMANCE IN CS1 USING THE COMPUTER TERMINOLOGY TEST}

The second aim of this research is to explore the efficacy of using the CTT to predict NNES students' academic performance in CS1 courses. For this purpose, the students' grades in the practical programming assessment, final exam and total score were collected at the end of the course. As the two universities have different grading systems, a grade normalisation was performed before the final analysis. Both universities use the same categories for grades (Excellent, Very Good, Good, Acceptable, and Fail), which were used to define a function to convert values between schemes for comparison.

Furthermore, the factor of proficiency in the English language needed to be controlled to eliminate its impact on the validity of the CTT. For this reason, the students' score in a standard English language test was used. The used English test scores range from 1 to 100 and are mapped to 6 language ability levels: beginner, elementary, intermediate, upper-intermediate, advanced, proficient [16]. The average English score for Chinese students is $\mu=55.2$ with standard deviation of 11.7, which place them between the intermediate to advanced levels. The average English score for Saudi students is $\mu=42.5$ with standard deviation of 11.8 , which means that the Saudi students' levels range from elementary to upper-intermediate.

Regression analyses were performed using the three academic measures as dependent variables and the CTT and English proficiency test scores as independent variables. In this experiment, the original version of the CTT was used (none of the test items was excluded). The main purpose of this analysis is to determine the efficacy of using the CTT to predict NNES students' academic performance in CS1 courses after controlling the students' proficiency in the English language.

Table 4 shows the result of regression analysis before and after controlling the English proficiency variable. The coefficient of determination $\left(r^{2}\right)$ is usually used to determine the proportion of the total variability of $Y$ that is accounted for or explained by $\mathrm{X}[19,39]$. 
In the same table, $\left(r^{2}\right.$ Change) represents the $r^{2}$ when the English language proficiency impact is removed.

Based on the analysis results, the ability of the CTT to predict the two groups of students' performance is different. However, the ability of the CTT to predict the final exam score for both groups is not very different $\left(r^{2}\right.$ Change $\left.=0.48,0.52, p<.001\right)$ for groups 1 and 2 respectively. The big difference is in the practical assignment score, where the $r^{2}$ Change is not statistically significant. Consequently, the difference in $r^{2}$ Change values for the practical assignment has been reflected in the $r^{2}$ Change values of the course results.

For group 1, which represents the Chinese students, $\left(r^{2}\right.$ Change $=$ .482 ), which means that $48 \%$ of the variability in total score can be predicted from the relationship with the CTT after removing the impact of the students' English proficiency. In the case of group 2, which represents the Saudi students, $66 \%$ of the variance in the total CS1 score can be explained or predicted by the CTT. Additionally, about $50 \%$ of the variance in the final exam scores for both groups can be correlated with their knowledge level of computer terminology regardless of their English proficiency level. Therefore, it can be said that the CTT test can be beneficial in predicting NNES students' academic performance in CS1 courses, particularly their academic performance in the final exam.

\begin{tabular}{lll|ll} 
& \multicolumn{3}{c}{ Computer Terminology Test } \\
\cline { 2 - 5 } & \multicolumn{2}{c}{ Group 1 $(n=92)$} & \multicolumn{2}{c}{ Group 2 $(n=59)$} \\
\cline { 2 - 5 } & $r^{2}$ & $r^{2}$ Change & $r^{2}$ & $r^{2}$ Change \\
\hline Practical Assignment & .140 & .112 & .409 & $.373^{*}$ \\
Final Exam & .511 & $.482^{*}$ & .604 & $.516^{*}$ \\
Course Result & .414 & $.381^{*}$ & .769 & $.660^{*}$ \\
\hline
\end{tabular}

* Statistically significant $(p<.001)$

Table 4: Regression Analysis Results

Moreover, a significant low-to-moderate positive correlation was found between CTT and the English test results $(r=0.381, p<$ .001). The obvious reason for this correlation is that at their core, both tests are language tests for the same language but from different perspectives. However, the CTT is more related to the content of CS1 courses and is considerably shorter and easier to administer than most available English proficiency tests.

\section{CONCLUSION}

In this paper, the responses of 150 NNES students on a CTT have been used to find evidence of the validity of the test. Based on this analysis, the test has an acceptable level of validity in all six aspects of test validity described by Messick [36]. The validity results are limited to NNES students and cannot be extended to native English speakers. Additionally, the test in this study has been provided through an online testing platform; accordingly, the impact of different testing methods on the test validity is not clear.

Moreover, this research investigated the efficacy of using the CTT to help in identifying NNES students who may struggle in CS1 courses because of English language difficulty. The results show the possibility of predicting about $50 \%$ of the academic performance in the final CS1 exam using CTT scores. The results of this research correspond with the findings of Keen and Etzkorn [23] that limited knowledge of computer terminology tends to have a negative impact on students' academic performance in programming courses.

In summary, this research provides evidence of the validity of the CTT based on the collected data. Additionally, the analysis performed in this study demonstrates the potential in using this test as a predictor for the NNES students' academic performance in CS1 courses, especially in predicting students' final exam results.

\section{LIMITATIONS AND FUTURE WORK}

It was planned to conduct the research based on traditional CS1 courses that adopt a face-to-face teaching method. However, as a result of COVID-19 restrictions in China and Saudi Arabia, both CS1 courses have been delivered online. The exact impact of the teaching and testing method (i.e., online or face-to-face) on the research result cannot be determined. While we believe that this change does not have a significant impact on the findings, further investigation into the impact of both teaching and testing methodologies on the observed correlations is needed.

The majority of the students in this study did not have experience in programming and had not attended programming courses prior to this course. Therefore, it was expected that they would not have knowledge of any computer terminology related to programming at the beginning of the course. Accordingly, the CTT was applied in the middle of the course after the students had had the opportunity to learn some of the programming basic concepts. However, the future plan is to run the CTT twice, with one test in the first week of the CS1 course and the other in the middle of the course. It is expected that this will provide more insights into what this test is measuring and how it can be used to help identify NNES students who may struggle in the CS1 course.

Moreover, this test was originally designed for NNES students, which justifies the selection of non-native English speakers as the sample in this research. However, including native English- speaking students could have helped in determining whether the native language makes a difference in performance. Therefore, future research may consider comparing native and non-native Englishspeaking students.

Future research is planned to investigate the use of this CTT along with other instruments to build a predictive model that identifies NNES students who may struggle in CS1 classes. Furthermore, the results of this research have highlighted the need for supporting resources and tools to help first-year CS students master computer terminology, especially NNES students. This is part of a larger ongoing research project that aims to improve CS1 courses for NNES students.

\section{REFERENCES}

[1] Suad Alaofi. 2020. The Impact of English Language on Non-Native English Speaking Students' Performance in Programming Class. In Proceedings of the 2020 ACM Conference on Innovation and Technology in Computer Science Education (Trondheim, Norway) (ITiCSE '20). Association for Computing Machinery, New York, NY, USA, 585-586. https://doi.org/10.1145/3341525.3394008

[2] Suad Alaofi and Seán Russell. 2021. Computer Terminology Test for Non-native English Speaking CS1 Students. In Proceedings of the 52nd ACM Technical Symposium on Computer Science Education. Association for Computing Machinery, New York, NY, USA, 1304-1304.

[3] Vahid Aryadoust, Hannah Ann Hui Tan, and Li Ying Ng. 2019. A Scientometric review of Rasch measurement: The rise and progress of a specialty. Frontiers in 
psychology 10 (2019), 2197.

[4] P Baghaei. 2008. The Rasch model as a construct validation tool. Rasch Measure ment Transactions 22, 1 (2008), 1145-1146.

[5] Purya Baghaei and Nazila Amrahi. 2011. Validation of a multiple choice English vocabulary test with the Rasch model. fournal of Language Teaching \& Research 2, 5 (2011)

[6] David Beglar. 2010. A Rasch-based validation of the Vocabulary Size Test. Language testing 27, 1 (2010), 101-118.

[7] Jia Bi. 2020. How large a vocabulary do Chinese computer science undergraduates need to read English-medium specialist textbooks? English for Specific Purposes 58 (2020), 77-89. https://doi.org/10.1016/j.esp.2020.01.001

[8] Maryna Bogachyk and Dmytro Bihunov. 2020. The Structural-Semantic Features of Computer Terms in English. Cognitive Studies/ Études cognitives 20 (2020).

[9] Trevor Bond. 2004. Validity and assessment: a Rasch measurement perspective. Metodología de las Ciencias del Comportamiento 5 (2004), 179-194.

[10] T Bond and C Fox. 2001. Applying the Rasch model. Mahwah, NJ: L.

[11] William J Boone and Kathryn Scantlebury. 2006. The role of Rasch analysis when conducting science education research utilizing multiple-choice tests. Science Education 90, 2 (2006), 253-269.

[12] Dennis Bouvier, Ellie Lovellette, John Matta, Bedour Alshaigy, Brett A Becker, Michelle Craig, Jana Jackova, Robert McCartney, Kate Sanders, and Mark Zarb. 2016. Novice programmers and the problem description effect. In Proceedings of the 2016 ITiCSE Working Group Reports. 103-118.

[13] Stephanie Buono and Eunice Eunhee Jang. 2021. The Effect of Linguistic Factors on Assessment of English Language Learners' Mathematical Ability: A Differential Item Functioning Analysis. Educational Assessment 26, 2 (2021), 125-144.

[14] Nell Dale. 2005. Content and emphasis in CS1. ACM SIGCSE Bulletin 37, 4 (2005), 69-73.

[15] Aintzane Doiz, David Lasagabaster, and Juan Manuel Sierra. 2012. Englishmedium instruction at universities: Global challenges. Multilingual matters.

[16] EF-Education. [n.d.]. English levels and English proficiency scores: EF SET https://www.efset.org/english-score/

[17] Education First. 2020. EF Standard English Test. https://www.efset.org/.

[18] Huda Gedawy, Saquib Razak, and Hanan Alshikhabobakr. 2019. The Effectiveness of Creating Localized Content for Middle School Computing Curriculum. In Proceedings of the 2019 ACM Conference on Innovation and Technology in Computer Science Education. 478-484.

[19] F Gravetter and L Wallnau. 2015. Statistics for the behavioral sciences . Cengage Learning.

[20] Philip J Guo. 2018. Non-native english speakers learning computer programming: Barriers, desires, and design opportunities. In Proceedings of the $2018 \mathrm{CHI}$ conference on human factors in computing systems. 1-14.

[21] Matthew Hertz. 2010. What do" CS1" and" CS2" mean? Investigating differences in the early courses. In Proceedings of the 41st ACM technical symposium on Computer science education. 199-203.

[22] Ed.D James Sick. 2009. Rasch Measurement in Language Education Part 4: Rasch Analysis Software Programs. , (p. 13 - 16) pages.

[23] Kevin J Keen and Letha Etzkorn. 2009. Predicting students' grades in computer science courses based on complexity measures of teacher's lecture notes. Fournal of Computing Sciences in Colleges 24, 5 (2009), 44-48.

[24] Truman Lee Kelley. 1927. Interpretation of educational measurements. (1927).

[25] Abdullah Aied Khuwaileh. 2010. IT terminology and translation: Cultural, lexicographic and linguistic problems. LSP fournal-Language for special purposes, professional communication, knowledge management and cognition 1, 2 (2010).

[26] Jane Knight. 2013. The changing landscape of higher education internationalisation - for better or worse? Perspectives: Policy and Practice in Higher Education 17, 3 (2013), 84-90. https://doi.org/10.1080/13603108.2012.753957

[27] Carl A Lager. 2006. Types of mathematics-language reading interactions that unnecessarily hinder algebra learning and assessment. Reading Psychology 27, 2-3 (2006), 165-204.

[28] John M. Linacre. 2021. A user's guide to Winsteps/Ministeps Rasch-Model programs, Program Manual 5.0.0.

[29] John M. Linacre. 2021. Winsteps. http://www.winsteps.com

[30] Ernesto Macaro, Samantha Curle, Jack Pun, Jiangshan An, and Julie Dearden 2018. A systematic review of English medium instruction in higher education. Language Teaching 51, 1 (2018), 36-76.

[31] Zunita Mohamad Maskor, Harun Baharudin, and Maimun Aqsha Lubis. 2018 Measurement validity and reliability of the productive vocabulary knowledge instrument for Arabic learners in Malaysian secondary schools. Advanced Science Letters 24, 5 (2018), 3423-3426.

[32] Raina Mason and Carolyn Seton. 2020. Assessing International Students: The Role of Cognitive Load. In Proceedings of the Twenty-Second Australasian Computing Education Conference. 160-166.

[33] Raina Mason and Carolyn Seton. 2021. Leveling the playing field for international students in IT courses. In Australasian Computing Education Conference. 138-146.

[34] Tim McNamara and Ute Knoch. 2012. The Rasch wars: The emergence of Rasch measurement in language testing. Language Testing 29, 4 (2012), 555-576.
[35] Samuel Messick. 1989. Validity. (3ed ed.). Macmillan, New York, 13-103.

[36] Samuel Messick. 1996. Validity and washback in language testing. Language testing 13, 3 (1996), 241-256.

[37] Daniel E Minshall. 2013. A Computer Science Word List. Master's thesis. Swansea University, Swansea.

[38] Yali Mu and Hao Bai. 2020. A Study on Influencing Factors of the Application Level of Computer in Chinese for International Students. In 2020 The 4th International Conference on Education and Multimedia Technology. 244-249.

[39] Robert R Pagano. 2012. Understanding statistics in the behavioral sciences. Cengage Learning.

[40] Georg Rasch. 1960. Studies in mathematical psychology: I. Probabilistic models for some intelligence and attainment tests. (1960).

[41] Kenneth D Royal. 2009. Making Meaningful Measurement in Survey Research: The Use of Person and Item Maps. Online Submission (2009).

[42] Ivan Ruby and Bojana Krsmanovic. 2017. Does learning a programming language require learning English? A comparative analysis between English and programming languages. In EdMedia+ Innovate Learning. Association for the Advancement of Computing in Education (AACE), 420-427.

[43] Judith Runnels. 2011. Evaluation of an achievement English vocabulary test using Rasch analysis. (2011)

[44] Judith Runnels. 2012. Using the Rasch model to validate a multiple choice English achievement test. International fournal of Language Studies 6, 4 (2012), 141-155.

[45] Chris R. Sawyer, Kurk Gayle, Andrew Topa, and William G. Powers. 2014. Listening Fidelity Among Native and Nonnative English-Speaking Undergraduates as a Function of Listening Apprehension and Gender. Communication Research Reports 31, 1 (2014), 62-71. https://doi.org/10.1080/08824096.2013.844119

[46] Judy Sheard, Angela Carbone, Selby Markham, Angas John Hurst, Des Casey, and Chris Avram. 2008. Performance and progression of first year ICT students. In Proceedings of the tenth conference on Australasian computing education-Volume 78. 119-127.

[47] Everett V Smith Jr. 2001. Evidence for the reliability of measures and validity of measure interpretation: a Rasch measurement perspective. Fournal of applied measurement (2001).

[48] Purwo Susongko. 2016. Validation of science achievement test with the rasch model. Jurnal Pendidikan IPA Indonesia 5, 2 (2016), 268-277.

[49] Edward W Wolfe and Everett V Smith Jr. 2007. Instrument development tools and activities for measure validation using Rasch models: part II-validation activities. Journal of applied measurement 8, 2 (2007), 204-234.

[50] Benjamin D Wright and Mark Stone. 1999. Measurement essentials. 


\section{A COMPUTER TERMINOLOGY TEST (WITH ANSWERS)}

Please select the correct terminology that matches the definition in each of the following questions:

1. A number system that only uses two digits -0 and 1
a. Binary
b. Octal
c. Hexadecimal

2. The process by which a computer carries out the instructions of a program.

a. Execution

b. Processing

c. Programming

3. A sequence of instructions that is continually repeated until a certain condition is reached.
a. Loop
b. Condition
c. Class

4. The spelling and grammar of instructions in a programming language.
a. Syntax
b. Semantics
c. Rules

5. Which of the following terms describes the type of value a variable has and the type of operations the variable can be used in:
a. data type
b. data structure
c. data classification

6. A single repetition of a process or set of instructions in a computer program.
a. Iteration
b. Initialization
c. Processing

7. The process of locating and removing computer program errors.
a. Debugging
b. Troubleshooting
c. Testing

8. The process of assigning an initial value to a variable.
a. Initialization
b. Assignment
c. Installation

9. Names that work like containers to hold numbers, phrases, or other important data used in several places in the code.
a. Variables
b. Functions
c. Holders

10. Type of expressions used to compare values and give only a true or false result.
a. Boolean
b. Binary
c. Pointer

11. The specific reserved words in a programming language.
a. Keywords
b. Variables
c. Identifiers

12. The name that the user gives to variable, function or class.
a. Identifier
b. Given-name
c. Forename

13. Commands that enable a program to take decisions about following one path or another:
a. control structure
b. control flow
c. control path

14. A whole number (not a fraction) that can be positive, negative, or zero:
a. integer
b. decimal
c. float

15. A named section of the code that performs a specific task:
a. function
b. parameters
c. package

16. Stop a running program:
a. Exit
b. Execute
c. delete

17. A set of instructions designed to perform a specific task:
a. algorithm
b. arithmetic
c. algebra

18. A punctuation mark used to separate elements:
a. comma
b. bracket
c. command

19. A variable that is used to keep track of anything that must be counted:
a. counter
b. tracker
c. connecter

20. The process of adding a value to a numerical value:
a. increment
b. decrement
c. inaccurate

21. Anything of, relating to, or containing numbers:
a. numeric
b. numbering
c. digit

22. A punctuation mark used to enclose arguments:
a. parentheses
b. semicolon
c. hashtag

23. A program that translates the source code (e.g. Java code) all at once into a language that computer can understand:
a. Compiler
b. Processor
c. Translator

24. A program that translates a source code (e.g. Python code) line-by-line into a language that computer can understand:
a. Interpreter
b. coder 
c. Translator

25. Is a piece of hardware that performs the calculations that run a computer:
a. Processor
b. calculator
c. operating system

26. A special format of organizing and storing data in computers:
a. data structure
b. storage
c. data type

27. An independent part of a program that performs one or more tasks:
a. module
b. software
c. code

28. The spaces or tabs at the beginning of a line of code:
a. Indentation
b. linear
c. blank first index:
a. Array/List
b. index
c. database

29. A data structure that contains a group of elements that are indexed according to a definite sequence with 0 being the

30. Inserting predefined module, package, or library into your code.
a. import/include
b. insert
c. export 